\title{
CMEARTICLE
}

\section{Clinics in diagnostic imaging (145)}

Jerome Irai Ezhil Bosco ${ }^{1}, \mathrm{MBBS}, \mathrm{MD}$, Albert SC $\underline{\mathrm{LoW}}^{2}$, MMed, FRCR, Damien MY $\underline{\operatorname{Tan}}^{3}$, MBBS, MRCP, Wilfred CG $\underline{P e h}^{1}$, FRCP, FRCR

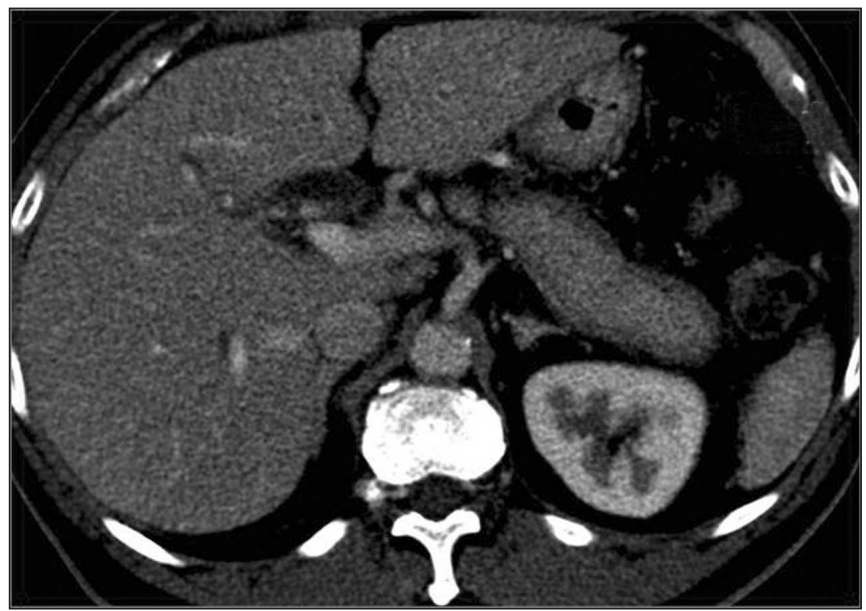

Fig. 1 Axial contrast-enhanced CT image (portal venous phase).

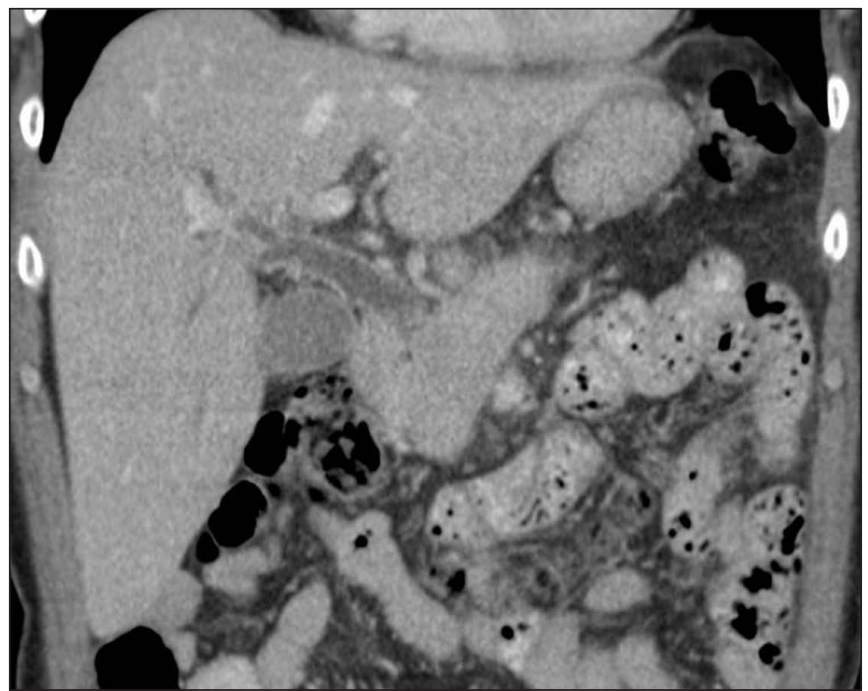

Fig. 2 Reconstructed coronal CT image.

\section{CASE PRESENTATION}

A 63-year-old man presented with painless jaundice for the past two weeks. He had a loss of appetite and significant weight loss of $5 \mathrm{~kg}$ over the past month. He had a history of diabetes mellitus, hypertension and hyperlipidaemia, and was a chronic smoker. On examination, the patient was icteric and wellhydrated, with normal vital parameters. Abdomen examination showed non-tender hepatomegaly. He was mildly anaemic (haemoglobin $12.8 \mathrm{gm} / \mathrm{dL}$ ), with altered liver function (total bilirubin $234 \mu \mathrm{mol} / \mathrm{L}$, albumin $37 \mathrm{~g} / \mathrm{L}$, total protein $69 \mathrm{~g} / \mathrm{L}$,

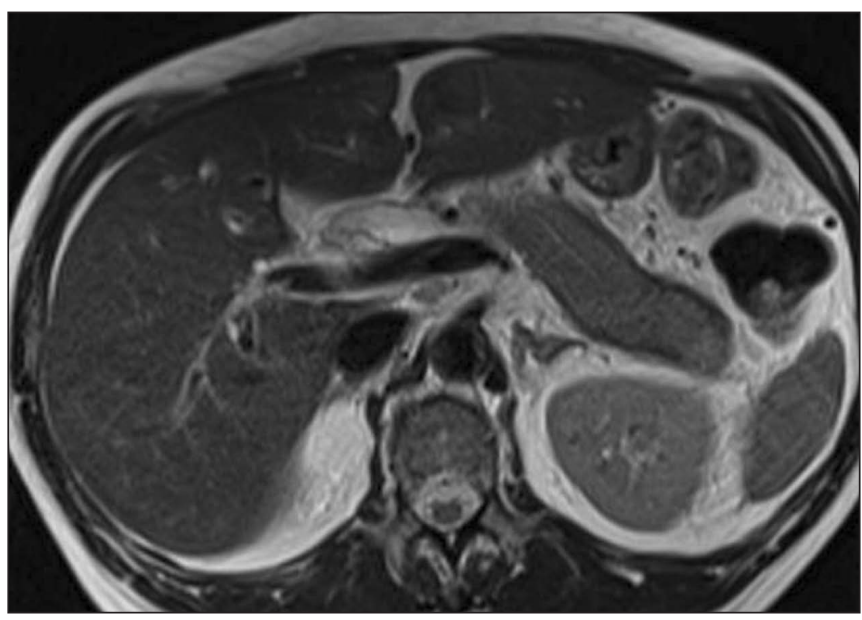

Fig. 3 Axial T2-W MR image

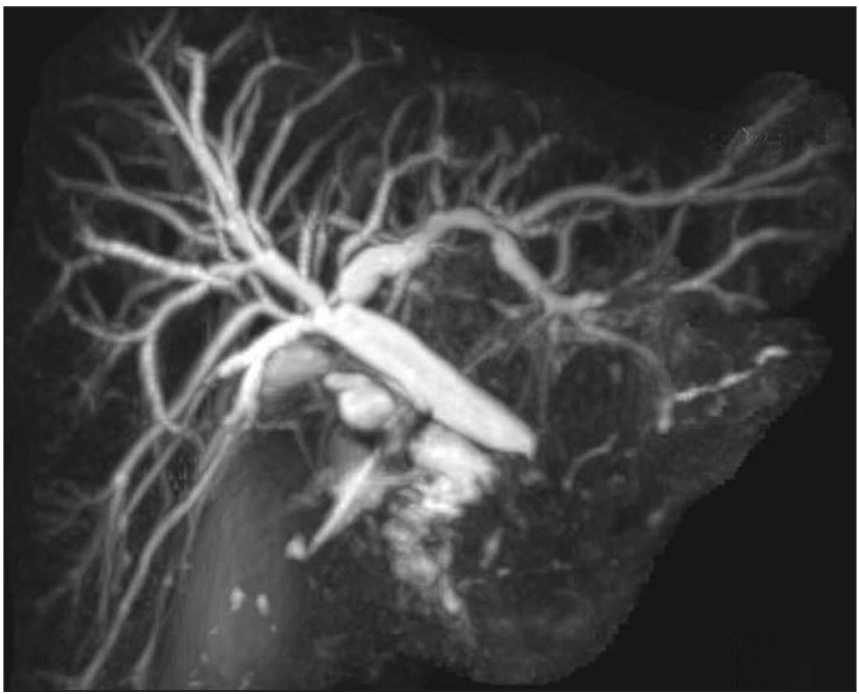

Fig. 4 MRCP image.

aspartate aminotransferase $210 \mathrm{U} / \mathrm{L}$, alanine aminotransferase $111 \mathrm{U} / \mathrm{L}$, alkaline phosphatase $411 \mathrm{U} / \mathrm{L}$, gamma-glutamyl transpeptidase $1830 \mathrm{U} / \mathrm{L}$ ). Serum amylase was normal at $94 \mathrm{U} / \mathrm{L}$. His renal function test, full blood count and coagulation profile were within normal limits. He underwent multiphasic contrastenhanced computed tomography (CT) of his abdomen (Figs. 1 \& 2) followed by magnetic resonance (MR) imaging (Fig. 3) and magnetic resonance cholangiopancreatography (MRCP) (Fig. 4). What do these images show? What is the diagnosis?

${ }^{1}$ Department of Diagnostic Radiology, Khoo Teck Puat Hospital, ${ }^{2}$ Department of Diagnostic Radiology, ${ }^{3}$ Department of Gastroenterology and Hepatology, Singapore General Hospital, Singapore

Correspondence: Prof Wilfred CG Peh, Professor and Head, Department of Diagnostic Radiology, Khoo Teck Puat Hospital, Alexandra Health, 90 Yishun Central, Singapore 768828. Wilfred.peh@gmail.com 


\section{IMAGE INTERPRETATION}

Contrast-enhanced CT showed a diffuse bulky pancreas, with loss of lobular margin (sausage-shaped) and no significant peripancreatic fat stranding. A characteristic hypodense rim (or pseudocapsule) around the pancreas was present (Fig. 1). No mass was seen in the biliary tract or pancreatic head. Biliary tract dilatation with sudden cut-off at the suprapancreatic region was present (Fig. 2). MR imaging showed the pseudocapsule as a T1- and T2-hypointense rim around the pancreas (Fig. 3). MRCP showed common bile duct dilatation with stricture at the suprapancreatic region, and pancreatic duct narrowing (Fig. 4).

\section{DIAGNOSIS}

Autoimmune pancreatitis (lymphoplasmacytic sclerosing pancreatitis).

\section{CLINICAL COURSE}

The patient underwent an endoscopic, ultrasonography-guided biopsy of the pancreas, which showed nonspecific inflammatory and fibrotic changes. The laboratory finding of raised immunoglobulin G subclass 4 (IgG4) antibodies (> $2.69 \mathrm{~g} / \mathrm{L}$ ) helped to confirm the diagnosis. The patient was started on high-dose steroids, which were slowly tapered off. He improved symptomatically, with repeat liver function tests showing significant improvement within a week and complete normalisation within five weeks. A follow-up, contrast-enhanced CT done one month after the patient was started on high-dose steroids showed normal appearance of the pancreas, including normal-sized gland and duct, with resolution of biliary dilatation (Fig. 5).

\section{DISCUSSION}

A type of chronic pancreatitis thought to be caused by an autoimmune mechanism was first described by Sarles et $\mathrm{al}_{,}{ }^{(1)}$ who termed it primary inflammatory sclerosis of the pancreas. The term autoimmune pancreatitis (AIP) came about when Yoshida et $\mathrm{al}^{(2)}$ reported a similar case and proposed the term, which is the currently accepted terminology for the condition. AIP, also known as lymphoplasmacytic sclerosing pancreatitis, is described as chronic inflammation of the pancreas due to a postulated autoimmune mechanism. ${ }^{(3)}$ It is associated with other autoimmune disorders, namely inflammatory bowel disease, primary sclerosing cholangitis, Sjogren syndrome, retroperitoneal fibrosis, diabetes mellitus, systemic lupus erythematous, vasculitis and thyroiditis.

AIP is an uncommon disease with characteristic imaging findings, which can help to differentiate it from pancreatic malignancy, which is a more common lesion. Differentiation is important because AIP responds dramatically to high-dose steroids and misdiagnosis could result in unnecessary surgical procedures, as seen in a previously reported case where a misdiagnosis led to major surgery, including Whipple's resection. ${ }^{(4,5)}$ AIP can be categorised as Type 1 or Type 2. Our patient had the more

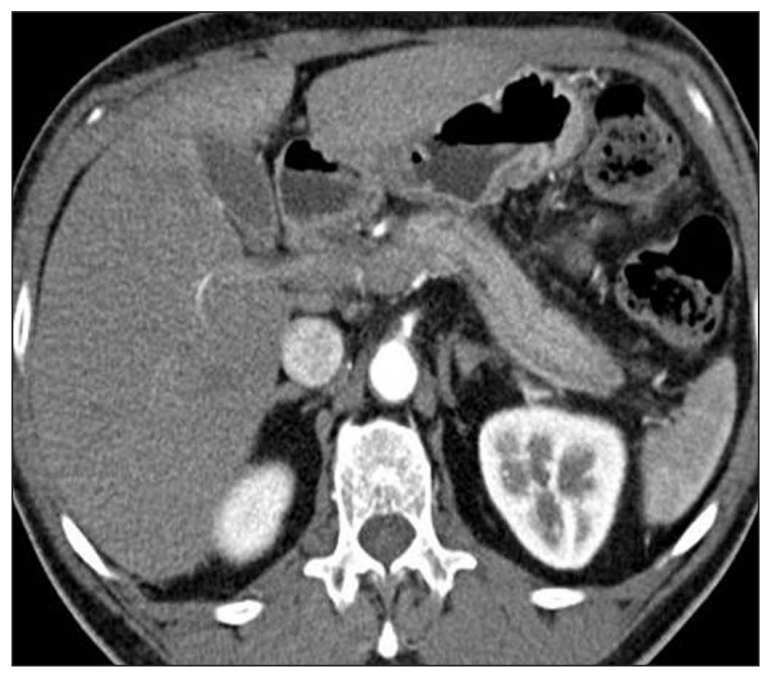

Fig. 5 Follow-up axial contrast-enhanced CT image (arterial phase) taken a month after high-dose steroid treatment shows a normal-sized pancreatic gland with interval resolution of the pancreatic duct narrowing.

common Type 1 disease, which is characteristically associated with raised IgG4 antibodies and male preponderance. ${ }^{(6)}$

The histological hallmark of AIP is periductal infiltration by lymphocytes and plasma cells, and granulocytic epithelial lesions with consequent destruction of the duct epithelium and venulitis. ${ }^{(7)}$ The presence of duct-centric inflammation is probably the most important histological feature in differentiating AIP from other inflammatory diseases of the pancreas. The clinical presentation is obstructive jaundice (stenosis of the intrapancreatic common bile duct in $63 \%-75 \%$ of patients), with or without mild abdominal pain, weight loss, and in elderly patients, recent-onset diabetes mellitus. The mainstay of treatment is high-dose steroid therapy, with reversible improvement of pancreatic morphology and function.

The Japan Pancreas Society ${ }^{(8,9)}$ has proposed the following diagnostic criteria for AIP:

1. Imaging results: Enlargement of the pancreatic parenchyma, ${ }^{(10)}$ with main pancreatic duct narrowing and irregular wall. Recent modifications include the presence of focal pancreatic mass and focal pancreatic duct stricture.

2. Laboratory data: Abnormally elevated levels of serum gamma globulin and/or lgG, or the presence of autoantibodies.

3. Histopathological findings: Fibrotic change with dense lymphoplasmacytic infiltration.

If criterion 1 is present with either criterion 2 or 3 , the diagnosis of AIP is deemed to be definitive. Two other diagnostic schemes proposed by the Korean Society of Pancreatology and Mayo Clinic are also in use. These two schemes use additional criteria, notably a response to steroids and the presence of extrapancreatic lesions.

AIP can show focal or diffuse involvement. In the former, a focal mass is typically noted, with pancreatic duct narrowing at the site of involvement and no or minimal upstream dilatation. Focal involvement is difficult to identify on imaging and it is often mistaken for malignancy. ${ }^{(5)}$ In diffuse involvement, the findings 


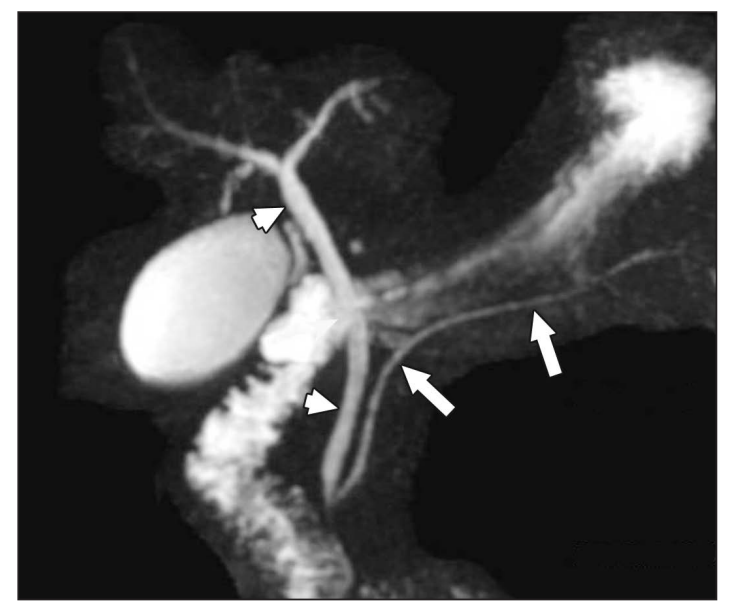

Fig. 6 MRCP image of another patient shows a normal common bile duct (arrowheads) and a normal pancreatic duct (arrows).

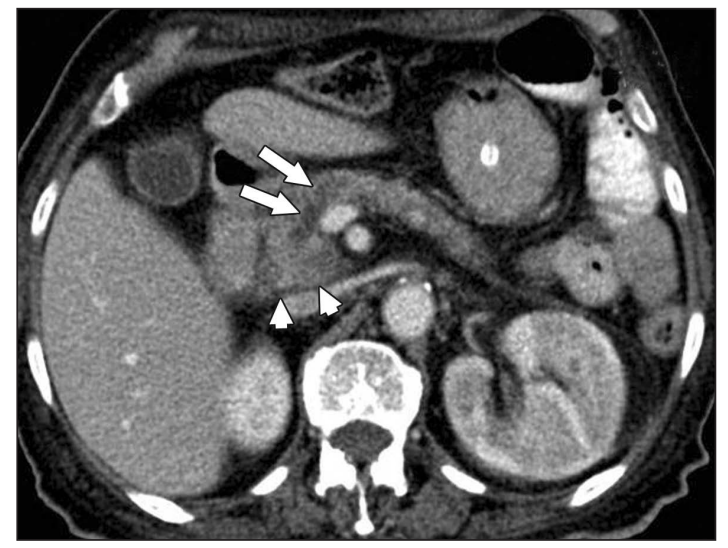

Fig. 7 Axial contrast-enhanced CT image of an 85-year-old diabetic and hypertensive woman being evaluated for obstructive jaundice shows a hypodense mass in the pancreatic head (arrowheads) with dilatation of the proximal pancreatic duct (arrows). These findings differentiate pancreatic malignancy from autoimmune pancreatitis, where the pancreatic duct proximal to the lesion is usually minimally dilated or normal. The patient was considered for palliative management due to her age and comorbidities.

- a bulky gland that is sausage-shaped due to the loss of normal lobular margins and no or limited peripancreatic fat stranding - are easier to interpret. The gland typically shows diminished signal intensity on T1-weighted MR images. The pancreatic duct is narrowed due to parenchymal oedema, and this is well appreciated on MRCP images. A normal MRCP image of another patient is shown for comparison (Fig. 6). A peripheral rim of a hypoattenuating halo on $\mathrm{CT}$ has been considered a classical finding in AIP. ${ }^{(10,11)}$ This is thought to represent inflammatory cell infiltration and is seen in up to $40 \%$ of patients with AIP. The rim is hypointense on both T1- and T2-weighted MR images, and shows delayed enhancement on contrast-enhanced MR images. On contrast-enhanced CT, the pancreas appears hypoattenuating as compared to the spleen, with delayed parenchymal enhancement on dynamic imaging. ${ }^{(12)}$ Enlargement of regional lymph nodes and biliary dilatation due to stenosis in the region of the pancreatic head, with smooth, beak-like narrowing in the distal common bile duct, are common extrapancreatic findings. ${ }^{(13)}$ Other extrapancreatic sites of involvement include the kidneys (present in about 35\% of patients), retroperitoneum and salivary glands.
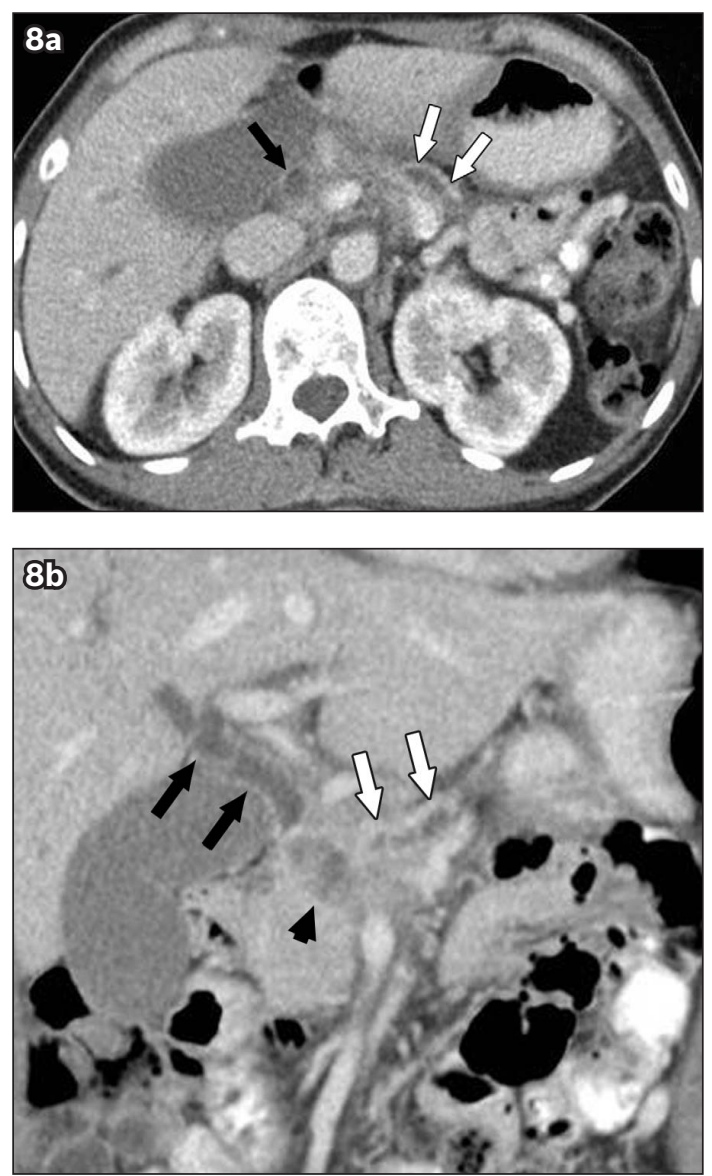

Fig. 8 (a) Axial and (b) reconstructed coronal contrast-enhanced CT images taken in the portal venous phase in a 54-year-old woman who presented with nonspecific vomiting shows a pancreatic head neoplasm (arrowhead) with the classical double duct sign - dilatation of the proximal pancreatic duct (white arrows) and common bile duct (black arrow) proximal to the tumour mass. In contrast, in autoimmune pancreatitis, the pancreatic duct proximal to the mass is usually normal in calibre. The malignancy was considered inoperable, and endoscopic retrograde cholangiopancreatography with stenting was offered to this patient.

The CT findings in pancreatic malignancy are a low-attenuating mass in both the hepatic and pancreatic phases of contrast study, significant dilatation of the pancreatic duct proximal to the mass, along with atrophy of pancreatic parenchyma and infiltration/ encasement of the adjacent vessels (namely the superior mesenteric artery, superior mesenteric vein, splenic vein and portal vein) (Figs. $7 \&$ 8). In AIP, on the other hand, there is diffuse or segmental narrowing of the main pancreatic duct with minimal or no dilatation proximal to the involved segment. Involvement of the adjacent vascular structures is uncommon in AIP, as is atrophy of pancreatic parenchyma proximal to the affected segment. ${ }^{(14,15)}$ Since delayed contrast enhancement of the affected segment in AIP is not seen in malignancy, it has also been mentioned to be a differentiating factor. ${ }^{(15)}$

\section{CONCLUSION}

In summary, care should be exercised when interpreting diagnostic images of male patients who present with a sudden onset of painless jaundice and associated autoimmune disease. While diffuse involvement of the pancreas gives a more classical picture (a sausage-shaped pancreas, with no significant fat 
stranding and a hypoattenuating rim on CT/hypointense halo on T1- and T2-weighted MR images), more diligence needs to be exercised in excluding malignancy for focal involvement.

\begin{abstract}
A 63-year-old man presented with painless jaundice, loss of appetite and significant weight loss. Cross-sectional imaging showed a diffusely enlarged pancreas, with no significant fat stranding and a hypodense rim on computed tomography, which appeared hypointense on T2-weighted magnetic resonance imaging. There was a narrowed pancreatic duct and features of common bile duct narrowing in the region of the pancreatic head. However, there was no obvious mass seen in the pancreatic head region. These features were classical of autoimmune pancreatitis with diffuse involvement of the gland. Laboratory investigation showed abnormal liver function and the classical sign of raised immunoglobulin $\mathrm{G}$ class 4 antibodies. The patient showed dramatic response to high-dose steroids, with resolution of both the laboratory and imaging abnormalities within one month. We discuss the classical imaging features of Type 1 autoimmune pancreatitis, an uncommon condition that needs to be differentiated from pancreatic malignancy.
\end{abstract}

Keywords: autoimmune pancreatitis, immunog/obulin G class 4 antibodies, lymphoplasmacytic sclerosing pancreatitis, pancreatic malignancy, sausageshaped pancreas

\section{REFERENCES}

1. Sarles H, Sarles JC, Muratore R, Guien C. Chronic inflammatory sclerosis of the pancreas-an autonomous pancreatic disease? Am J Dig Dis 1961; 6:688-98.

2. Yoshida K, Toki F, Takeuchi T, et al. Chronic pancreatitis caused by an autoimmune abnormality. Proposal of the concept of autoimmune pancreatitis. Dig Dis Sci 1995; 40:1561-8.

3. Okazaki K, Chiba T. Autoimmune related pancreatitis. Gut 2002; 51:1-4.

4. Servais A, Pestieau SR, Detry O, et al. Autoimmune pancreatitis mimicking cancer of the head of the pancreas: case report of 2 cases. Acta Gastroenterol Belg 2001; 64:227-30.

5. Sahani DV, Kalva SP, Farrell J, et al. Autoimmune pancreatitis: imaging features. Radiology 2004; 233:345-52.

6. Zen Y, Bogdanos DP, Kawa S.Type 1 autoimmune pancreatitis. Orphanet J Rare Dis 2011; 6:82.

7. Klöppel G, Lüttges J, Sipos B, Capelli P, Zamboni G. Autoimmune pancreatitis: pathological findings. JOP 2005; 6(1 Suppl):97-101.

8. Chari ST, Smyrk TC, Levy MJ, et al. Diagnosis of autoimmune pancreatitis: the Mayo Clinic experience. Clin Gastroenterol Hepatol 2006; 4:1010-6.

9. Okazaki K, Uchida K, Fukui T, Matsushita M, Takaoka M. Recent advances in autoimmune pancreatitis. Gastroenterol Hepatol (N Y) 2008; 4:417-26.

10. Van Hoe L, Gryspeerdt S, Ectors N, et al. Nonalcoholic duct-destructive chronic pancreatitis: imaging findings. Am J Roentgenol 1998; 170:643-7.

11. Kim KP, Kim MH, Kim JC, et al. Diagnostic criteria for autoimmune chronic pancreatitis revisited. World J Gastroenterol 2006; 12:2487-96.

12. Irie $\mathrm{H}$, Honda $\mathrm{H}$, Baba $\mathrm{S}$, et al. Autoimmune pancreatitis: $\mathrm{CT}$ and MR characteristics. AJR Am J Roentgenol 1998; 170:1323-7.

13. Bodily KD, Takahashi N, Fletcher JG, et al. Autoimmune pancreatitis: pancreatic and extrapancreatic imaging findings. AJR Am J Roentgenol 2009; 192:431-7.

14. Kamisawa T, Egawa N, Nakajima H, et al. Clinical difficulties in the differentiation of autoimmune pancreatitis and pancreatic carcinoma. Am J Gastroenterol 2003; 98:2694-9.

15. Takahashi N, Fletcher JG, Hough DM, et al. Autoimmune pancreatitis: differentiation from pancreatic carcinoma and normal pancreas on the basis of enhancement characteristics at dual-phase CT. AJR Am J Roentgenol 2009; 193:479-84. 


\section{SINGAPORE MEDICAL COUNCIL CATEGORY 3B CME PROGRAMME} (Code SMJ 201304B)

Question 1. Regarding autoimmune pancreatitis:

(a) The most important differential for this condition is malignancy.

(b) It is also termed lymphoplasmacytic sclerosing pancreatitis.

(c) Type I is commonly seen in young females.

(d) Presenting complaints include severe abdominal pain with abdominal masses.

Question 2. Regarding the diagnostic criteria for autoimmune pancreatitis:

(a) The Japan Pancreas Society diagnostic criteria include elevated levels of serum gamma globulin and/or immunoglobulin G.

(b) The Korean Society of Pancreatology guidelines does not include treatment response as a definitive criteria.

(c) Imaging criteria include focal mass or diffuse involvement.

(d) The characteristic histopathological finding is duct-centric inflammation.

Question 3. Regarding the management of autoimmune pancreatitis:

(a) It responds dramatically to high-dose steroids.

(b) There is usually irreversible damage to pancreatic morphology and function.

(c) Histopathological tests are necessary for diagnosis.

(d) Newer criteria used for diagnosis include treatment response to steroid, and extrapancreatic lesions.

Question 4. Regarding computed tomography findings in autoimmune pancreatitis:

(a) In the focal type of involvement, a sausage-shaped pancreas has been described.

(b) A peripheral rim of a hypoattenuating halo is characteristically described in the diffuse type of involvement.

(c) On contrast imaging, the pancreas is hyperattenuating as compared to the spleen.

(d) The distal common bile duct shows a smooth, beak-like narrowing near the pancreatic head.

Question 5. Regarding magnetic resonance imaging findings in autoimmune pancreatitis:

(a) A hypointense halo is seen on T1- and T2-weighted images.

(b) The pancreatic duct is usually dilated in the diffuse type of involvement.

(c) The pancreas shows low signal intensity on T1-weighted images.

(d) In pancreatic malignancy, the pancreatic duct proximal to the mass is dilated, as compared to the focal type of involvement in autoimmune pancreatitis.

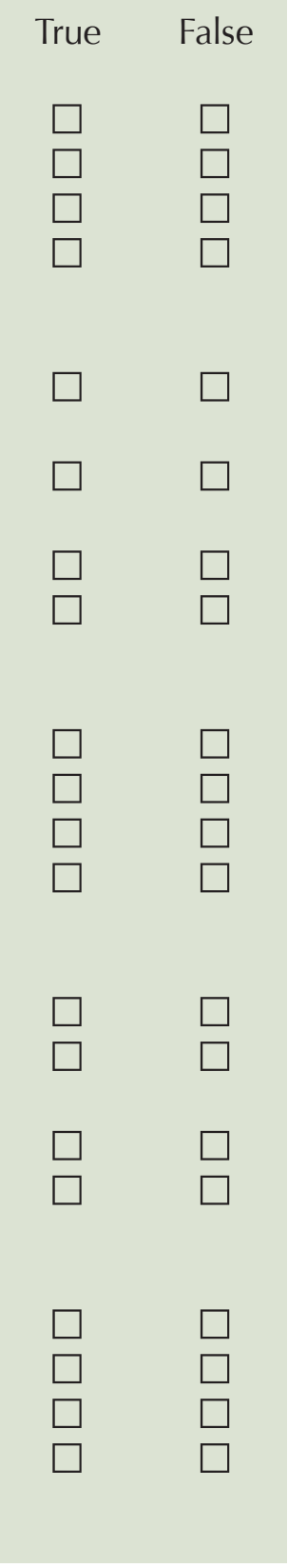

\footnotetext{
Doctor's particulars:

Name in full

MCR number

Specialty:

Email address

(1) Log on at the SMJ website: http://www.sma.org.sg/cme/smj and select the appropriate set of questions. (2) Select your answers and provide your name, email address and MCR number. Click on "Submit answers" to submit.

RESULTS:

(1) Answers will be published in the SMJ June 2013 issue. (2) The MCR numbers of successful candidates will be posted online at www.sma.org.sg/cme/smj by 27 May 2013. (3) All online submissions will receive an automatic email acknowledgement. (4) Passing mark is $60 \%$. No mark will be deducted for incorrect answers. (5) The SMJ editorial office will submit the list of successful candidates to the Singapore Medical Council. (6) One CME point is awarded for successful candidates.

Deadline for submission: (April 2013 SMJ 3B CME programme): 12 noon, 20 May 2013.
} 